Document downloaded from:

http://hdl.handle.net/10251/180288

This paper must be cited as:

Vergara, M.; Rezaei, A.; Schramm, G.; Rodríguez-Alvarez, MJ.; Benlloch Baviera, JM.; Nuyts, J. (2021). 2D feasibility study of joint reconstruction of attenuation and activity in limited angle TOF-PET. IEEE Transactions on Radiation and Plasma Medical Sciences. 5(5):712-722. https://doi.org/10.1109/TRPMS.2021.3079462

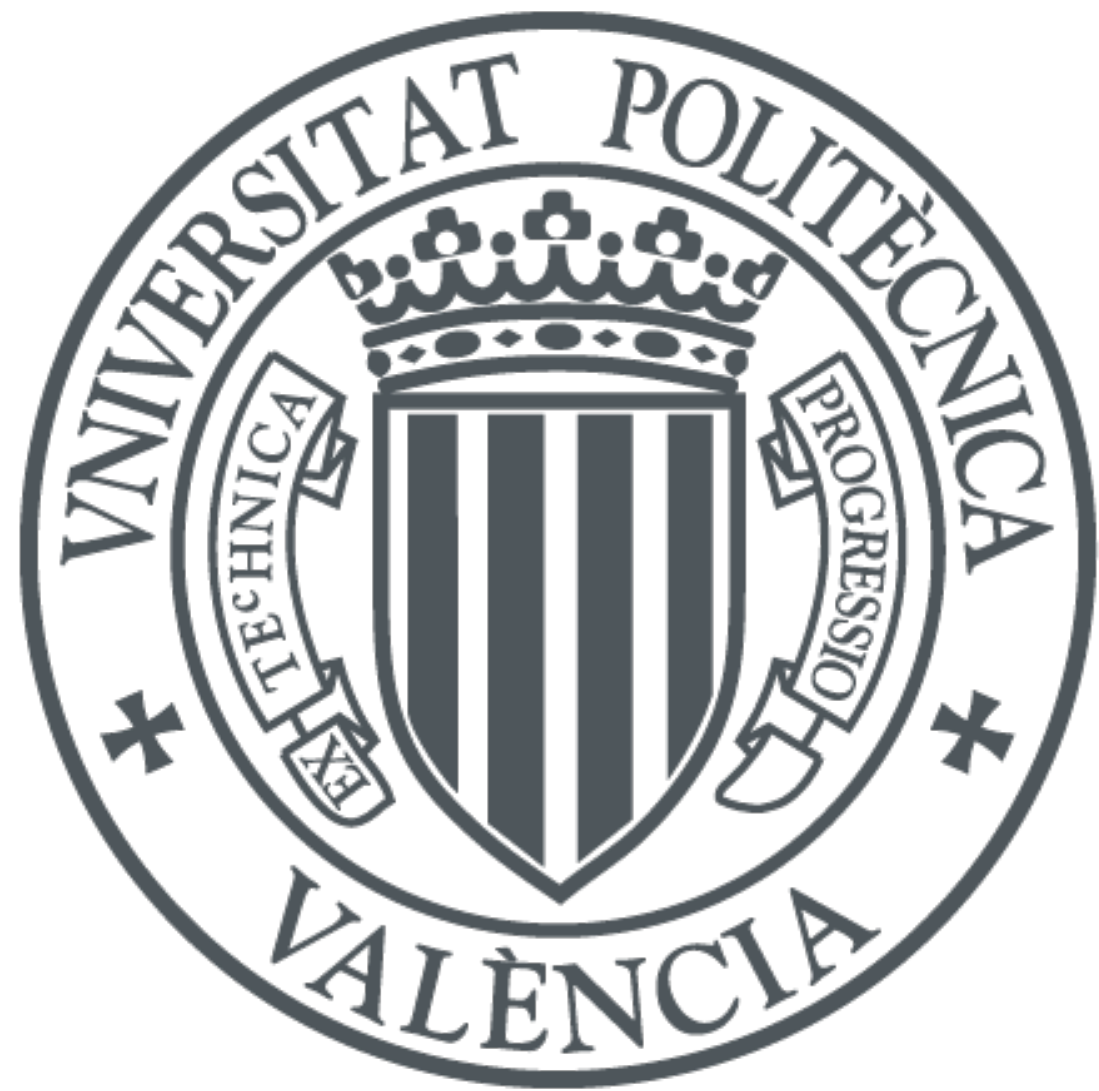

The final publication is available at

https://doi.org/10.1109/TRPMS.2021.3079462

Copyright Institute of Electrical and Electronics Engineers

Additional Information 


\title{
2D feasibility study of joint reconstruction of attenuation and activity in limited angle TOF-PET
}

\author{
Marina Vergara*, Ahmadreza Rezaei, Georg Schramm, Maria Jose Rodriguez-Alvarez, \\ Jose Maria Benlloch Baviera and Johan Nuyts
}

\begin{abstract}
Several research groups are studying organdedicated limited angle positron emission tomography (PET) systems to optimize performance-cost ratio, sensitivity, access to the patient and/or flexibility. Often open systems are considered, typically consisting of two detector panels of various sizes. Such systems provide incomplete sampling due to limited angular coverage and/or truncation, which leads to artefacts in the reconstructed activity images. In addition, these organ-dedicated PET systems are usually stand-alone systems, and as a result, no attenuation information can be obtained from anatomical images acquired in the same imaging session. It has been shown that the use of time-of-flight information reduces incomplete data artefacts and enables the joint estimation of the activity and the attenuation factors. In this work, we explore with simple 2D simulations the performance and stability of a joint reconstruction algorithm, for imaging with a limited angle PET system. The reconstruction is based on the so-called MLACF (Maximum Likelihood Attenuation Correction Factors) algorithm and uses linear attenuation coefficients in a known-tissueclass region to obtain absolute quantification. Different panel sizes and different time-of-flight (TOF) resolutions are considered. The noise propagation is compared to that of MLEM reconstruction with exact attenuation correction (AC) for the same PET system. The results show that with good TOF resolution, images of good visual quality can be obtained. If also a good scatter correction can be implemented, quantitative PET imaging will be possible. Further research, in particular on scatter correction, is required.
\end{abstract}

Index Terms-Time-of-Flight, limited angle, sinogram truncation, dedicated systems, PET imaging, interior problem.

\section{INTRODUCTION}

$\mathbf{M}$ ost of the commercially available PET (positron emission tomography) systems are intended for whole-body imaging and are combined with CT (computerized tomography) or MR (magnetic resonance) imaging capabilities in order to obtain anatomical information, which is also used to produce the attenuation image [1] for attenuation correction during PET image reconstruction. In recent years, organdedicated PET systems have been proposed as alternatives to whole-body scanners, aiming at systems that are less expensive, require less space and/or provide easier patient access, higher resolution and/or better sensitivity [2]-[8]. Some of

*M. Vergara is with the Department of Imaging and Pathology, Division of Nuclear Medicine, KU Leuven, Belgium and Instituto de Instrumentación para Imagen Molecular Centro Mixto CSIC-Universitat Politècnica de València, Valencia, Spain (e-mail: marina.vergara@uzleuven.be).

J.M. Benlloch Baviera and M.J. Rodriguez-Alvarez are with Instituto de Instrumentación para Imagen Molecular Centro Mixto CSIC-Universitat Politècnica de València, Valencia, Spain, A. Rezaei, G. Schramm and J. Nuyts are with the Department of Imaging and Pathology, Division of Nuclear Medicine, KU Leuven, Belgium. these systems, such as those consisting of two opposing detector panels, acquire only incomplete tomographic data, due to limited angular coverage. In addition, many of these systems are not combined with a modality providing anatomical images (CT or MR) and therefore, the conventional approaches to attenuation correction cannot be applied.

A voxel is said to have "limited angular coverage" if the set of acquired LORs intersecting the voxel does not satisfy the local Tuy condition [9], [10], and therefore the intensity of the voxel cannot be reconstructed exactly. A PET system will be said to provide only limited angle data, if the above holds for all voxels inside the field of view of that system. In 2D, a PET system acquires limited angle data if the sinogram covers less than $180^{\circ}$. Because reconstruction from limited angle data does not have a unique solution, reconstructed images will almost always suffer from artefacts. It has been shown that in PET, the availability of time-of-flight information reduces these limited angle artefacts [3], [11], [12].

Apart from that, the PET data will be called "truncated", if for at least some of the parallel projections covered by the system, the object is truncated in the projection. In 2D, this means that the sinogram has been truncated at one or both sides. If a system, suffering from truncation at both sides in all projections, has a region inside the field of view for which complete angular coverage was obtained, then this system is said to face the interior problem [13]. In [14] it was proven that if the intensities are known inside a small subregion of the interior region, then the entire interior region can be reconstructed exactly from the truncated data. With this convention, a system always suffers from truncation if it has limited angular coverage, but not the other way around.

If PET images are reconstructed without attenuation correction, the images are distorted by artefacts and the reconstructed activity values suffer from large negative, object and position dependent bias. Fortunately, when TOF information is available, joint reconstruction algorithms can be used to estimate the attenuation sinogram ${ }^{1}$ from the emission data up to a global constant [15] for all the LORs where activity is present, provided that the spread of the tracer is wider than the TOF resolution. This constant can be determined if prior knowledge about attenuation values (or the activity) is available [16]-[21].

Another effect hindering quantification is the contribution of scattered photons to the acquired PET data. In conventional non angular-limited PET imaging, a scatter estimate is

\footnotetext{
${ }^{1}$ Throughout the text attenuation sinogram will refer to the projection of the attenuation image $(-\log (a))$, as defined in section II-B, while $a$ will be called attenuation factors.
} 


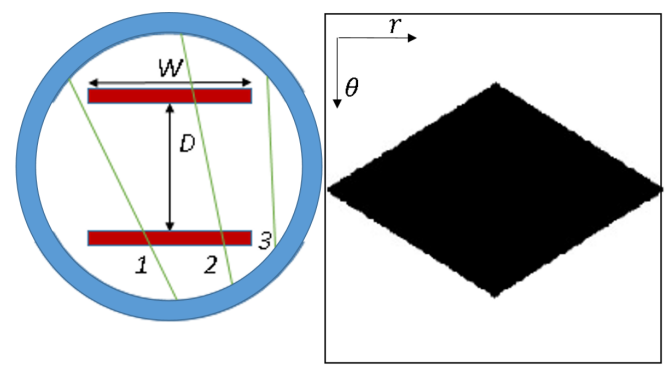

Fig. 1. Left: The PET ring (blue) and the pair of flat panels (red) of size $W$ separated by distance $D$. Only LORs that hit both flat panels (LOR 2) will be considered. This is not the case for LOR 1 (only hits one panel) and LOR 3 (that does not hit any of them). Right: Sensitivity sinogram mask of the system; $r$ and $\theta$ are the radial and angular indexes, respectively.

computed from a preliminary activity image (obtained without scatter correction) and the attenuation image provided by the anatomical imaging system. This scatter estimate is then used to reconstruct a better activity image, and iterating this procedure has been shown to converge. For a stand-alone system, such an attenuation image is not available but other approaches can be followed as described in Section IV-C.

In this work we consider the problem of jointly reconstructing the activity image and the attenuation factors from TOF-PET data suffering from limited angular coverage or truncation. We propose an approach based on the MLACF algorithm of [22]. For limited angle data, it is not guaranteed that all sinogram pixels are affected by the same constant, but below we show that in many cases this will be the case. To determine the value of the constant, the attenuation image is reconstructed from the estimated attenuation factors. The problem is studied only in $2 \mathrm{D}$, enabling us to perform many simulations and reconstructions in a short time, which is very useful for a first exploration. As shown below, images of good visual quality can be obtained for PET with high TOF resolution, even for systems providing very limited angular coverage and severe truncation. If in addition a good scatter correction method can be implemented, quantitative PET imaging will be possible. We provide some preliminary results indicating that scatter correction should be possible, but further research will be required.

\section{METHODS}

\section{A. System design}

In order to examine the effect of limited angular coverage in TOF-PET image reconstruction, we use a 2D simulation of a circular PET system (figure 1). We consider limited angle effects similar to those seen by a pair of flat panels of size $W$, separated by distance $D$, by setting to zero the sensitivity value of all the LORs that are not seen by both flat panels, as illustrated in figure 1. Examples of selected and rejected LORs are shown as well. With this system modelling approach, systems with different angular coverage can be compared avoiding confounding effects due to differences in sampling. The only difference between the systems is in which fraction of the complete set of LORs they sample.

\section{B. Joint reconstruction}

In order to exploit the TOF information in the joint estimation of activity and attenuation, two algorithms have been proposed: Maximum Likelihood reconstruction of Attenuation and Activity (MLAA) [16] and Maximum Likelihood Attenuation Correction Factors (MLACF) [22]. Here, the MLACF algorithm is applied because of its faster execution time.

In TOF PET, the expected count $\bar{y}_{i t}$ for a certain line of response (LOR) $i$ and TOF-bin $t$ can be written as:

$$
\bar{y}_{i t}^{(m, n)}=a_{i}^{(m)} p_{i t}^{(n)}+r_{i t} \quad \text { with } \quad p_{i t}^{(n)}=\sum_{j} c_{i j t} \lambda_{j}^{(n)}
$$

where $p_{i t}$ is the unattenuated TOF projection of the activity image $\lambda$ for LOR $i$ in TOF-bin $t, a_{i}$ is the attenuation factor along the LOR $i, c_{i j t}$ is the sensitivity of the measurement bin $(i, t)$ for activity in voxel $j$ in the absence of the attenuation, $r_{i t}$ is an additive contribution made by randoms and/or scatter and the superscripts denote iteration indices.

Then the MLACF algorithm is given by:

$$
\begin{aligned}
a_{i}^{(m+1)}= & a_{i}^{(m)}+\frac{a_{i}^{(m)}}{p_{i}^{(n)}} \sum_{t} p_{i t}^{(n)} \frac{y_{i t}-\bar{y}_{i t}^{(m, n)}}{\bar{y}_{i t}^{(m, n)}} \\
\lambda_{j}^{(n+1)}= & \lambda_{j}^{(n)}+\frac{\lambda_{j}^{(n)}}{\sum_{i} c_{i j} a_{i}^{(m+1)}} \times \\
& \sum_{i t} c_{i j t} a_{i}^{(m+1)} \frac{y_{i t}-\bar{y}_{i t}^{(m+1, n)}}{\bar{y}_{i t}^{(m+1, n)}}
\end{aligned}
$$

where $n$ and $m$ describe the iteration number on the activity and on the attenuation correction factors (ACFs) respectively, $p_{i}=\sum_{t} p_{i t}$, and $c_{i j}=\sum_{t} c_{i j t}$. This algorithm is very similar to the regular MLEM algorithm [23], except that in every iteration, the ACFs are iteratively updated using (2) as described in [22].

We have used the additive implementation of the MLACF algorithm because it has better numerical stability than the multiplicative form. We also found the use of a matched TOF and non-TOF projector to be crucial for artefact-free reconstructions.

If the TOF resolution is small compared to the spatial extent of the activity distributions, then the derivatives of the attenuation sinogram are determined by the TOF data [15]. Integration produces the attenuation sinogram up to the integration constant. Therefore, if two sinogram points can be connected by an integration path that has activity everywhere along the path, then the two points have the same integration constant. As illustrated in figure 1 and figure 11 below, the panel sizes considered here preserve a single patch of LORs. For tracers like FDG, which accumulate almost everywhere in the body, there will be integration paths with activity between all non-zero sinogram points. For such tracers, the integration constant will be the same for all LORs that have seen activity, despite the gaps produced by the angular limitation. This constant term corresponds to a constant scale of the activity image.

If a quantitative image is needed, this scale factor must be determined. To do so, we propose to reconstruct an image 
from the attenuation factors and use prior information in image space (namely the known tissue attenuation in a certain region) to obtain the constant. To reconstruct the attenuation image we propose the MLTR algorithm [24]:

$$
\mu_{j}^{(n+1)}=\mu_{j}^{(n)}+\frac{\sum_{i} l_{i j} \frac{\bar{y}_{i}-r_{i}}{\bar{y}_{i}}\left(\bar{y}_{i}-y_{i}\right)}{\sum_{i} l_{i j} \sum_{k} l_{i k}\left(\bar{y}_{i}-r_{i}\right)\left(1-\frac{y_{i} r_{i}}{\bar{y}_{i}^{2}}\right)}
$$

where $\mu_{j}$ is the reconstructed linear attenuation coefficient in voxel $j, \overline{y_{i}}=p_{i} \exp \left(-\sum_{j} l_{i j} \mu_{j}^{(n)}\right)+r_{i}, r_{i}=\sum_{t} r_{i t}$ is the non-TOF randoms and/or scatter additive contribution and $l_{i j}$ is the intersection length of LOR $i$ and pixel $j$. Equation 4 uses the measured activity $y_{i}=\sum_{t} y_{i t}$ as transmission scan and the unattenuated forward projection of the reconstructed activity $p_{i}=\sum_{t} p_{i t}$ as the blank scan. The TOF-PET activity reconstruction provides a good outline of the body for most tracers. To improve the MLTR reconstruction, the body contour was determined by thresholding the activity image, and attenuation outside the body contour was forced to zero.

Here, the MLTR algorithm uses only the non-TOF data. When maximizing the TOF likelihood function with respect to the attenuation and with additive contamination present, the attenuation is updated using the TOF measurements/expressions [18], however the attenuation reconstruction is essentially a non-TOF reconstruction. As a result of not having TOF information available, the attenuation image will be more sensitive to missing projection angles than the activity image.

In order to determine the scale factor, agreement of some reconstructed attenuation coefficient values with the known attenuation coefficient of soft tissues at $511 \mathrm{keV}$ was imposed [20], [25], [26]. A region composed mostly of soft tissue was identified in the image and the mean attenuation coefficient in that region was computed. The region was determined by thresholding the central region of the image, keeping the values greater than the median over the region. Given the ratio of the correct tissue attenuation coefficient value to the extracted mean attenuation value $(\beta)$, the following expression was used to estimate $\gamma$, the factor by which the activity image (and therefore the blank scan) had to be scaled.

$$
\sum_{i} c_{i j} \gamma \lambda_{j} e^{-\mu \beta L} \simeq \sum_{i} c_{i j} \lambda_{j} e^{-\mu L} \rightarrow \gamma \simeq\left(e^{\mu L}\right)^{\beta-1}
$$

The chosen value for the attenuation coefficient was $\mu=$ $0.0957 \mathrm{~cm}^{-1}$ and for the length it was $L=28.3 \mathrm{~cm}$ hence $e^{\mu L}=15$. The blank scan was then rescaled with the factor $\gamma$, the attenuation image with $\beta$ and a new MLTR-iteration was computed. This sequence of MLTR reconstruction and rescaling was repeated until $0.99<\gamma<1.01$, which typically happens after a few iterations. Note that the relation between the attenuation image and the transmission sinogram is nonlinear, which is why MLTR iterations are required to correctly propagate the effect of rescaling the activity with $\gamma$ into the attenuation image.

\section{Experiments}

In this section, the performance of the 2D PET system for scanning a heart phantom (figure 2) is studied for different

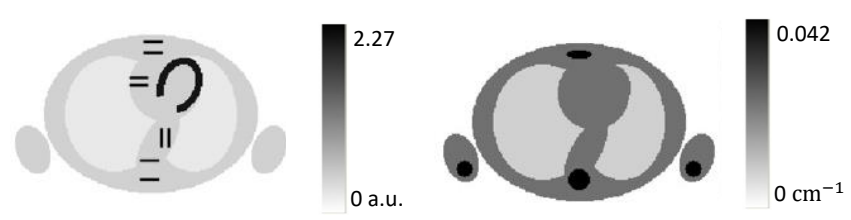

Fig. 2. Heart phantom emission (left) and attenuation (right) images

system parameters, including: full or limited angular coverage, sinogram truncation corresponding to panels having a width $W$ of 20 or $50 \mathrm{~cm}$, a TOF resolution of $250 \mathrm{ps}$ or $60 \mathrm{ps}$ FWHM and presence or absence of a scatter contribution. When limited angular coverage was modeled, it was equivalent to that of two panels at a distance of $30 \mathrm{~cm}$ and a width of 20 or $50 \mathrm{~cm}$.

Four small Defrise phantoms were added to the heart phantom. In 3D, a Defrise phantom consists of two (or more) parallel disks [27], and with the appropriate orientation, it is a very challenging object for limited angle tomography. In $2 \mathrm{D}$, the phantom reduces to two parallel rods (figure 2). These phantoms have been added with, from top to bottom, distances of 16, 10, 10 and $30 \mathrm{~mm}$ between the rods. Three of them are oriented horizontally to challenge the reconstruction, the fourth one is oriented vertically to show its near perfect reconstruction. In figure 3 , the amount of missing data is visualized with back-projections from identity sinograms (i.e. all sinogram pixels were set to 1 ).

The simulated activity and attenuation images had 270 x 270 pixels and an isotropic pixel size of $2 \mathrm{~mm}$. The simulations were computed using matched TOF and nonTOF projectors (ensuring that the non-TOF sinograms were equal to the summation of the corresponding TOF sinograms over their TOF bins). The TOF-PET data were organized in sinograms with 270 radial elements and 270 projection angles over $\pi$, with 37 TOF-bins of 100 ps and 127 TOF-bins of $30 \mathrm{ps}$ for the $250 \mathrm{ps}$ and $60 \mathrm{ps}$ FWHM TOF-resolution configurations, respectively. A uniform sinogram was added to the measurements to simulate a $50 \%$ randoms to primary ratio. The simulated emission data were then contaminated with Poisson noise generating 200 different noise realizations with $8.5 \cdot 10^{5}$ simulated counts in the untruncated activity sinogram. Those parameters were chosen having in mind an experimental cardiac TOF-PET system that is being developed by the $\mathrm{i} 3 \mathrm{~m}$ group in Valencia [28].

The images were reconstructed using 20 iterations, where the attenuation was updated 3 times for each update of the activity and no ordered subsets were used. The reconstructed images were post-smoothed with a $4 \mathrm{~mm}$ FWHM Gaussian kernel. The initialization of the algorithm was done using a uniform attenuation sinogram and a uniform image, both of value 1 , inside the field of view. Once an activity reconstruction was obtained from the MLACF algorithm, the body contour was defined by thresholding the activity reconstructed image. This was later used in the MLTR reconstruction to force 

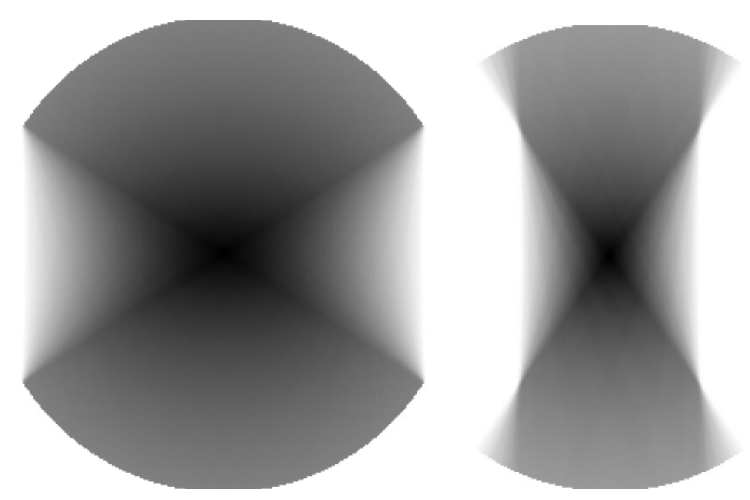

Fig. 3. Back-projection of an identity sinogram for two different panel widths: $50 \mathrm{~cm}$ (left) and $20 \mathrm{~cm}$ (right)

the attenuation outside the body contour to zero, as explained in Section II-B.

In the $20 \mathrm{~cm}$ configuration, heavy sinogram truncation in the radial direction is present, as the object is wider than the detector. To (somewhat artificially) separate the effects due to limited angular coverage from those due to truncation, we considered for each system also a version with the same truncation but with full angular coverage. One way to obtain this gap-less sinogram would be to rotate the two detector panels around the patient during the acquisition. In the following, we will refer to the system with limited-angle coverage as "open configuration" and to the one with full angular coverage as "closed configuration".

For a quantitative analysis of the reconstructed images, root mean square (RMS) values were computed for the difference image between the activity ground truth and the re-scaled MLACF reconstructed image. To provide a reference, also the RMS values of the difference between the ground truth activity image and a 20-iterations activity reconstruction with perfect AC was computed. Both values were calculated using the following equation:

$$
\mathrm{RMS}=\frac{1}{n} \sqrt{\sum_{j \in \mathrm{ROI}}^{n}\left(\lambda_{j}^{\text {ground truth }}-X_{j}\right)^{2}}
$$

where $X \in\left\{\lambda^{\text {re-scaled MLACF }}, \lambda^{\text {perf.atten MLEM }}\right\}$ activity reconstructions. These values were calculated for a circular ROI around each of the Defrise phantoms.

For the scatter simulation study, a scatter sinogram was generated. It was produced by convolving the trues sinogram with a 3D Gaussian with a FWHM of $120 \mathrm{~mm}$ in radial direction, 0.43 radians in angular direction and $94 \mathrm{~mm}$ in TOF direction. After smoothing, the scatter sinogram was multiplied with a scatter to trues ratio of $70 \%$. In the final sinogram, the ratio of scatters to prompts was 0.31 . The line profile of the central row of both TOF-integrated sinograms can be seen in figure 4.

In the experiments below, randoms were always simulated and corrected for during the reconstructions. Scatter was not simulated unless stated otherwise, and no scatter correction was applied.

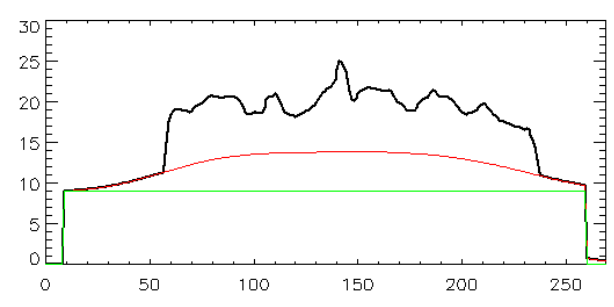

Fig. 4. TOF-integrated prompts (black), scatter and randoms (red) and randoms (green) line profiles along the central row
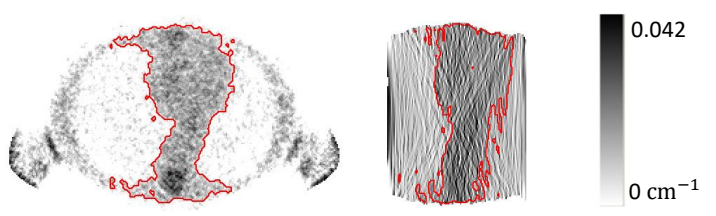

Fig. 5. In red: Thresholded soft-tissue region for the one noise realization simulation with the $50 \mathrm{~cm}$ closed (left) and the one noise realization in the $20 \mathrm{~cm}$ open configuration (right) configuration system operating at $250 \mathrm{ps}$ FWHM TOF resolution. Only the inner part of the attenuation image is selected to avoid truncation artifacts.

To analyze the noise characteristics, the re-scaled MLACF activity was compared to the MLEM activity reconstruction (20 iterations) using the exact attenuation factors for attenuation correction. This analysis was performed for one configuration: the closed system with 250 ps TOF resolution and $\mathrm{W}=50 \mathrm{~cm}$. The noisy sinograms were generated by varying the total number of counts to obtain different noise levels. 100 noise realizations were simulated. The pixel noise was quantified in two different regions of interest, by computing the average pixel standard deviation and dividing that by the mean of the region. This was done for a region containing the entire image and for a region containing the central third in which we apply the soft tissue prior information. This noise analysis was repeated after post-smoothing the images with a 2D Gaussian with $4 \mathrm{~mm}$ FWHM.

\section{RESULTS}

For the aforementioned open and closed configurations, simulations without and with a scatter contribution were performed, for two different TOF resolutions and two different panel sizes.

Figure 5 shows the thresholded noisy attenuation image, where the mean value of the selected pixels is driven to the known attenuation of tissue. To avoid contributions from the regions affected by truncation artefacts, only the central part of the image was used (1/3 of the image was ignored at the left and likewise at right).

\section{A. Noise propagation in re-scaled MLACF}

In figure 6, a comparison between the ratio of the standard deviation over the mean for different number of simulated counts can be seen for both activity reconstructions: MLEM 

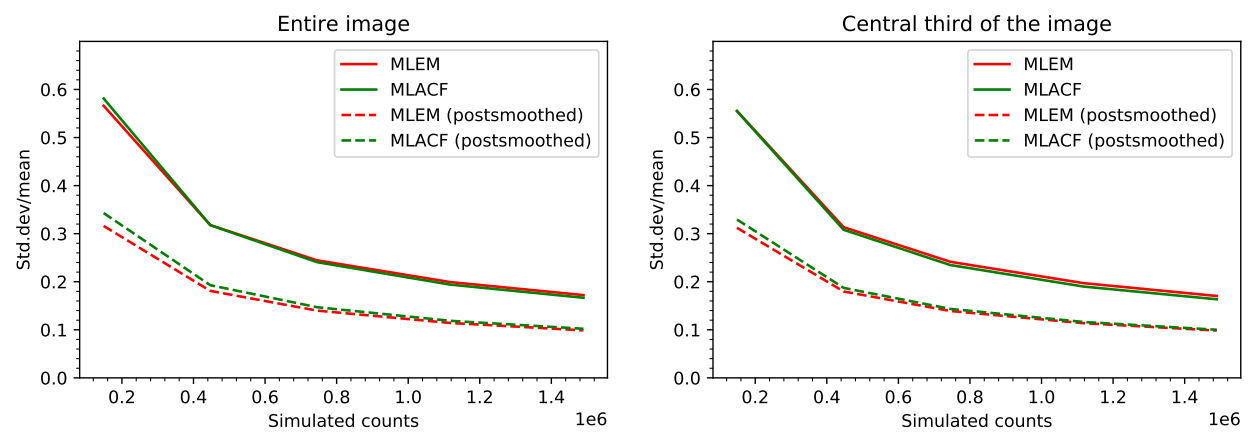

Fig. 6. Comparison of the noise in re-scaled MLACF images and images reconstructed with MLEM with perfect AC, for the closed PET system with 250 TOF resolution. The plots show the standard deviation divided by the mean for two regions: the entire image (left) and the central third of the image (right). These results have been computed from 100 noise realizations.

(with perfect $\mathrm{AC}$ ) and re-scaled MLACF. This analysis was done for the closed configuration with $250 \mathrm{ps}$ TOF resolution and $\mathrm{W}=50 \mathrm{~cm}$. The similarities of the plots show that both algorithms have a similar noise behavior.

\section{B. Simulations without scatter}

In this section, the results of scatter-free simulations are shown, for open and closed system configuration with both panel widths and for TOF resolutions of $250 \mathrm{ps}$ and $60 \mathrm{ps}$ FWHM.

Figure 7 shows the results of reconstructions from 200 noise realizations for the system corresponding to a detector panel size $W=50 \mathrm{~cm}$. Figure $7 \mathrm{a}$ shows the mean reconstruction images, their difference with the ground truth, one of the noisy reconstructions and the standard deviation image computed from the noise realizations. Some line profiles along a row through the apex of the heart are shown in figure $7 \mathrm{~b}$. Because the wall is oriented horizontally near the apex, the reconstruction of this part of the heart suffers most from limited angle artefacts in the open systems. These profiles also show that the mean images obtained are similar to the images obtained with reconstruction from noiseless data. The two narrowest horizontal Defrise phantoms are poorly reconstructed in the open configuration, but this is solved if the TOF resolution is improved to $60 \mathrm{ps}(9 \mathrm{~mm})$. As expected, the vertical Defrise phantoms are in all cases well reconstructed. The absolute differences between the reconstructions and ground truth images (also post-smoothed with a $4 \mathrm{~mm}$ gaussian) are small, except for the regions near the arms. As is typical for reconstruction from Poisson corrupted data, the standard deviations in the activity images are higher in pixels with higher activity.

Figure 8 shows the corresponding results for the PET system corresponding to the smaller detector panels with $W=20 \mathrm{~cm}$. The results of the activity reconstruction in the central part are comparable with the ones for the $W=50 \mathrm{~cm}$ panels, but for the open systems, the activity outside the central region has not contributed to the measurement and is therefore not reconstructed. The attenuation images for the closed systems show a good reconstruction of the interior region, but suffer from limited data artefacts in the rest of the image. For the open systems, the attenuation images suffer from limited angle artefacts (mainly a vertical blurring) everywhere.

RMS values of activity reconstructions and MLEM (using the perfect AC) were computed to be in a range of $[0.005,0.02]$ with a background activity of 0.4. In figure 9 bar plots comparing all the configurations are shown for each Defrise phantom. They quantitatively show that the performance of the re-scaled MLACF algorithm is close to that of MLEM with perfect $\mathrm{AC}$ as they both have similar differences from the ground truth, and these differences are small compared to the background activity. Similar results (shown in figure 10) are also obtained for the $50 \mathrm{~cm}$ and $250 \mathrm{ps}$ open and closed configurations.

In both configurations, the differences between the activity reconstructions are small in the central part of the phantom and they increase moving away from the center. This is clearly visible in the profiles: for the closed configuration, the profiles of the $250 \mathrm{ps}$ and $60 \mathrm{ps}$ reconstructions are quite similar, but for the open configuration some overshoots can be seen close to the edge of the plates.

In figure 10 we compare the mean (over noise realizations) activity reconstructions for both re-scaled MLACF and MLEM with perfect $\mathrm{AC}$ for the $250 \mathrm{ps}$ and $50 \mathrm{~cm}$ panels size in the case of complete tomography (closed) and limited angle (open) configurations. Figure 11 shows the estimated attenuation factors sinograms. This illustrates that for this activity distribution, all estimated attenuation factors are connected and therefore share the same constant, as explained above [14].

\section{Simulation with scatter}

In this section, the effect of the scattered coincidences is considered in the simulations but ignored during reconstruction. The aim was to verify if preliminary reconstructions obtained without any scatter correction are at least stable and of reasonable visual quality.

Activity and attenuation images were computed for a $2 \mathrm{D}$ simulation in the presence of scatter. The reconstruction results are presented in figures 12 and 13 for $W=50 \mathrm{~cm}$ and $W=20 \mathrm{~cm}$, respectively. The inclusion of scatter in the measurement without correcting for it during reconstruction produced the expected overestimation in the reconstructed activity images. The pixel standard deviation computed from 


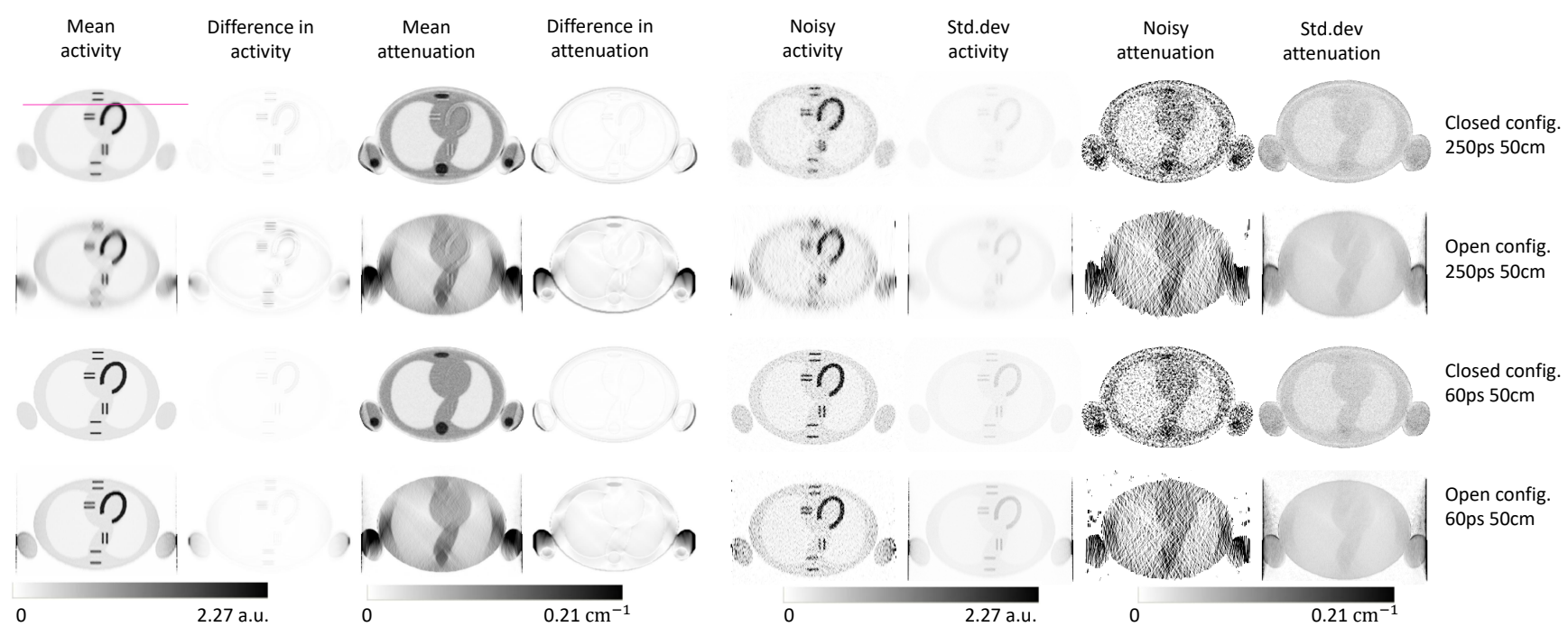

(a)
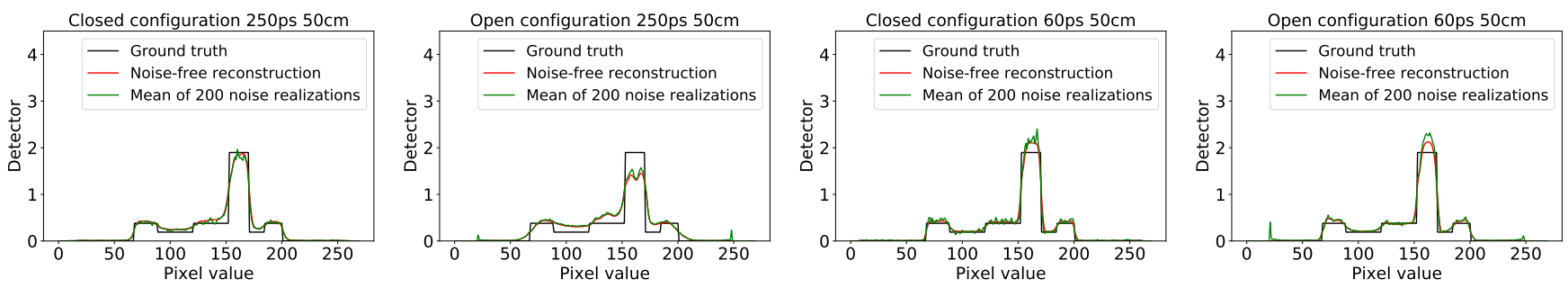

(b)

Fig. 7. Results from 200 noise realisations for $W=50 \mathrm{~cm}$ without scatter contribution, (from top to bottom): closed configuration with 250 ps, open configuration with $250 \mathrm{ps}$, closed configuration with $60 \mathrm{ps}$ and open configuration with $60 \mathrm{ps}$. In (a) and (from left to right): mean of the 200 noise realizations for the activity reconstruction, difference of this mean image with the ground truth, mean attenuation reconstruction, difference of this mean image with the ground truth, first noise realization of the activity, standard deviation for the 200 noise realizations of the activity, first noise realization of the attenuation and standard deviation of the attenuation. In (b) the line profiles of the activity image along a row through the apex of the heart (shown in (a) in purple) of the ground truth (black), the mean of 200 noise realizations (green) and the noiseless reconstructed image (red) for (from left to right) 250 ps closed configuration, $250 \mathrm{ps}$ open configuration, $60 \mathrm{ps}$ closed configuration and $60 \mathrm{ps}$ open configuration.

the 200 noise realisations has a similar magnitude as in the scatter-free simulation.

\section{DISCUSSION}

In this work, joint reconstructions of the activity and attenuation were done using a re-scaled MLACF approach. This algorithm was chosen instead of MLAA because of its faster convergence. MLACF is faster than MLAA, because estimating the attenuation factors requires fewer computations per iteration and converges much faster than estimating the attenuation image. As shown in [22], the attenuation factor estimation even reduces to a very fast non-iterative calculation if there are no scatter and randoms contributions. Consequently, most of the MLACF calculations are dedicated to estimating the activity image, and good convergence is obtained in fewer iterations than with MLAA. Once the attenuation factors are obtained, the attenuation image can be reconstructed with MLTR. Compared to the joint reconstruction during MLAA, this MLTR reconstruction of the attenuation image is more effective, because it can make use of a good activity estimate (obtained close to convergence). However, in case prior information is included to improve the image in the MLTR approach, an additional MLEM reconstruction of the activity would be required to propagate that information into the final activity image. In MLAA, that information would propagate automatically.

The reconstruction of the attenuation image is needed if quantification and/or scatter correction is required. In that case, the scale factor can be determined by imposing a-priori known attenuation coefficient values to regions in the attenuation image [25]. When the scale factor is obtained, the activity image can be re-scaled accordingly, no new activity reconstruction is required. For these 2D simulations, a good value of the scale factor could be obtained, despite the presence of limited angle artefacts in the attenuation images from the open systems and despite the presence of randoms.

\section{A. Noise propagation in re-scaled MLACF}

As shown in figure 6, the proposed method is stable and its noise characteristics are very similar to those of MLEM with exact AC, despite the fact that MLACF estimates more parameters from the same sinogram data. As shown in [22] 


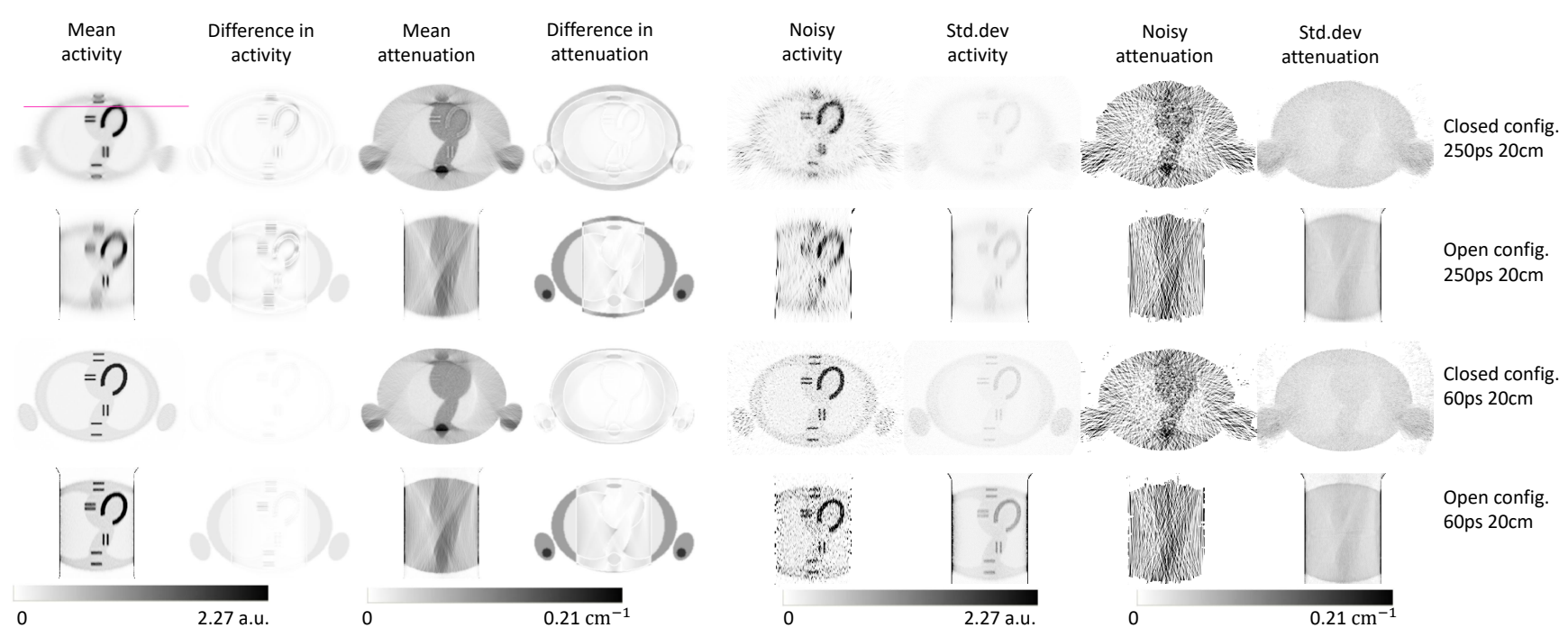

(a)
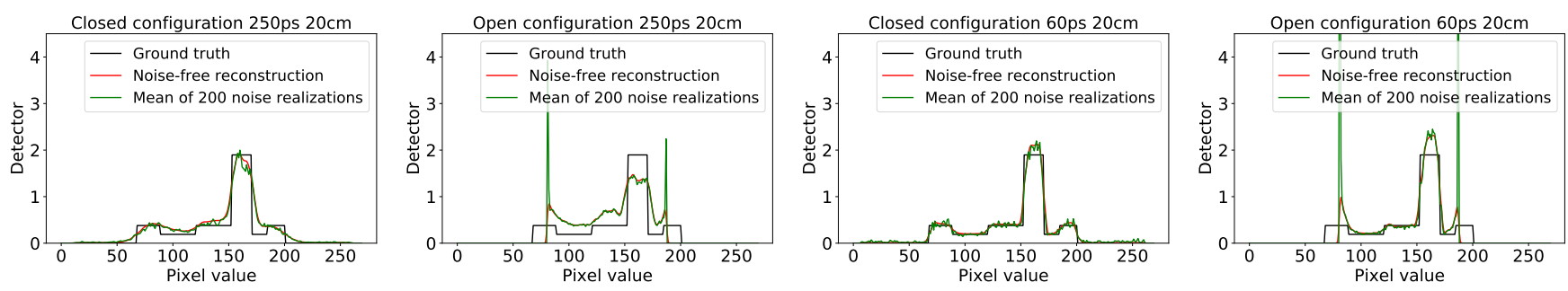

(b)

Fig. 8. Same results as in figure 7 for $W=20 \mathrm{~cm}$ without including scatter contribution.

for MLACF in complete tomography, MLACF has a slower convergence than MLEM, which present a lower bias and a higher variance in comparison to MLACF at the same number of iterations. This is shown as well in figure 10, where the reconstructed images shows good agreement among themselves in the presence of noise. However, there still are small differences, which are originated as a result of the non exactly matched convergence levels between MLEM and regular MLACF.

\section{B. Simulations without scatter}

In general, the images are worse for the smaller panels and for the open systems, because of the increased amounts of missing data. When large detector panels are used, (almost) all of the activity in the object contributes to the measurement. In contrast, for small panels, only a relatively small part of the activity is covered within the field of view. Limited angular coverage and truncation are strongly related and the distinction between the two depends on the exact definitions of the truncation and limited angle problems. With the definition used in this paper, the "closed" systems only suffer from truncation, whereas the "open" systems suffer from both truncation and limited angular coverage. For the closed system with 50 $\mathrm{cm}$ panels, the truncation is very limited, only part of the arms extend beyond the field of view and nearly artefact-free reconstructions are obtained. For the open system with 50 $\mathrm{cm}$ panels, the images are reconstructed from limited angle data. The effect of that is clearly revealed by the Defrise phantoms. The horizontal Defrise phantoms, and in particular the two narrow ones, are poorly reconstructed when the TOF resolution is $250 \mathrm{ps}$, because the system has no projection data that "have seen" the cold region between the hot rods. The vertical Defrise phantom is reconstructed accurately, because the two rods appear well separated in the acquired vertical projections. When the TOF resolution is improved to $60 \mathrm{ps}$, it provides a spatial resolution of $9 \mathrm{~mm}$ along the LORs. This enables the system to detect the cold region between the rods and to obtain accurate reconstruction of the Defrise phantoms.

In the quantitative analysis, the values of the RMS with respect to the ground truth were calculated around each Defrise phantom, as shown in figure 9. The RMS for all the $60 \mathrm{ps}$ configurations in each phantom are lower than the ones at 250 ps, showing that an improvement of the TOF resolution helps improving the quantitative activity estimation in MLACF. It is interesting to notice as well that in the horizontal Defrise phantoms (figure 9) the RMS values of the closed configurations and $50 \mathrm{~cm}$ panel size are generally lower than their open configurations and $20 \mathrm{~cm}$ counterparts, showing that the system performance improves when the truncation is less aggressive (in the case of $50 \mathrm{~cm}$ panel size) and when the tomography is complete (in the closed configurations). For 


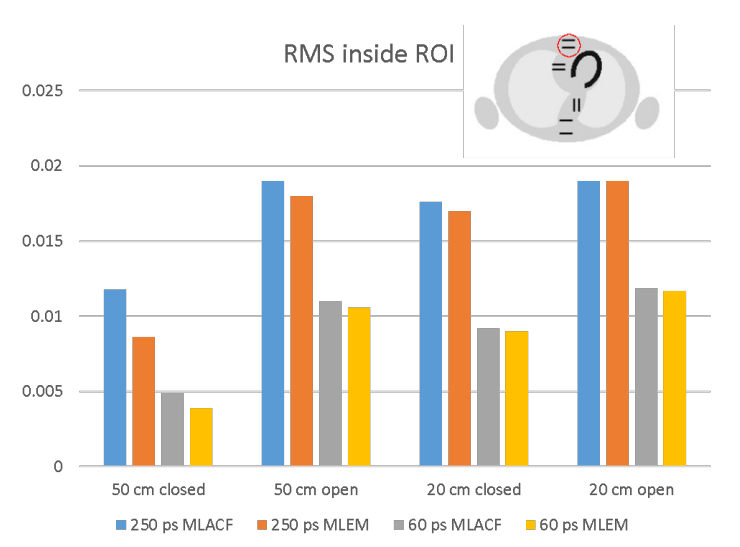

(a)

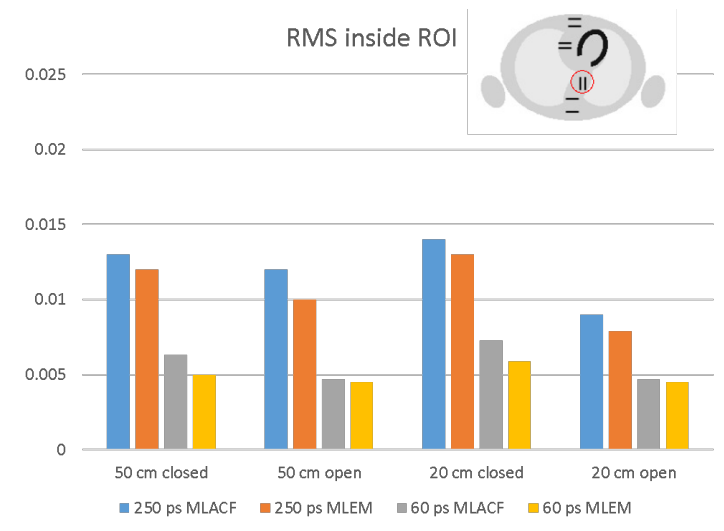

(c)

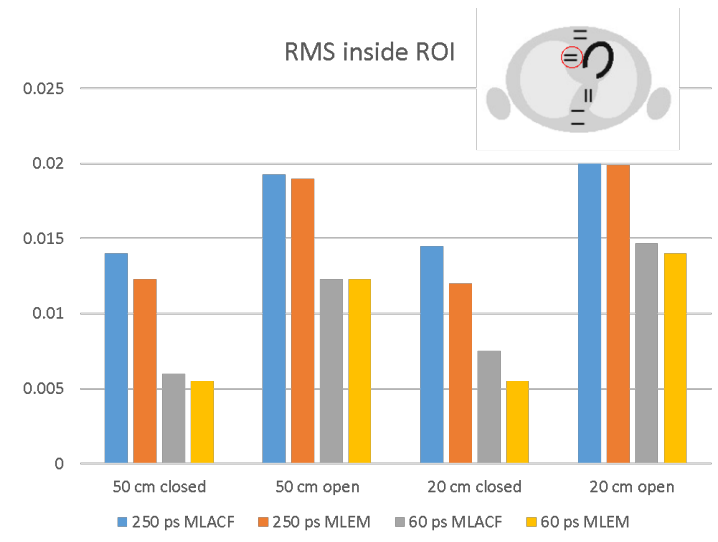

(b)

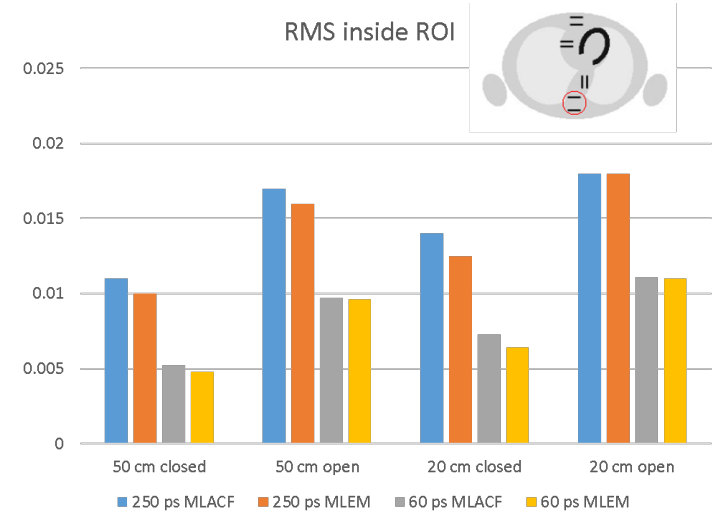

(d)

Fig. 9. Root mean squared (RMS) values calculated for different ROIs (in red) around each of the Defrise phantoms for the differences between ground truth and re-scaled MLACF activity reconstruction and MLEM with perfect AC. The background and the Defrise phantoms have a contrast ratio of 1:5, with an activity of 0.4 in the background and 2 in the rods.
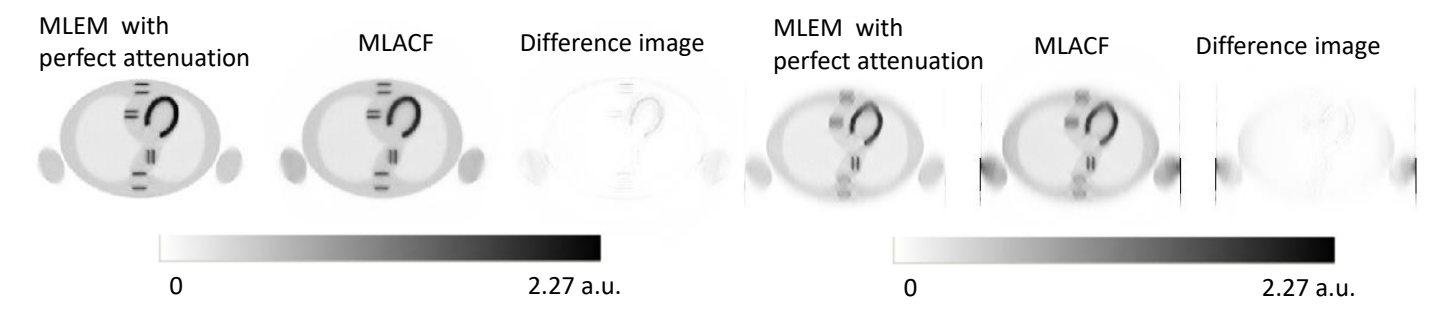

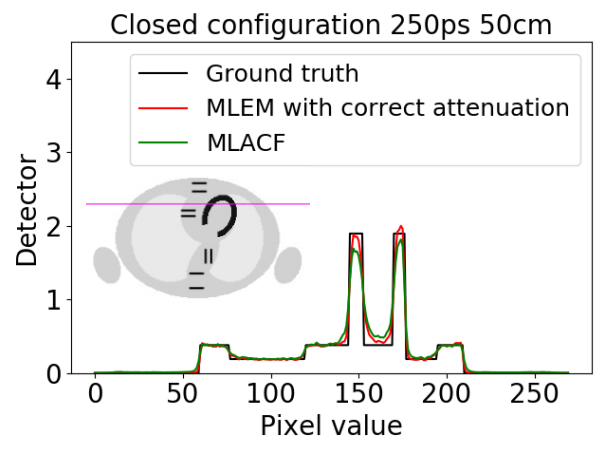

(a)

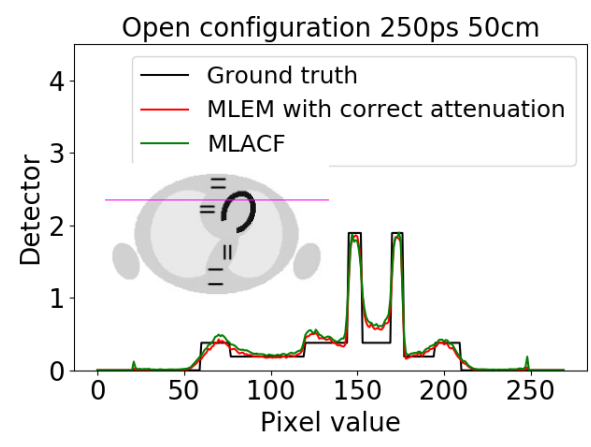

(b)

Fig. 10. from left to right: (a) Mean of the noise realizations of the activity reconstructed images from perfect attenuation MLEM, re-scaled MLACF, and the difference image. Bottom: Activity profiles along the line shown in the picture. Results are for the closed configuration, $50 \mathrm{~cm}$ panel size and 250 ps TOF resolution. (b): Same results as in (a) for open configuration, $50 \mathrm{~cm}$ panel size and $250 \mathrm{ps}$ TOF resolution. 

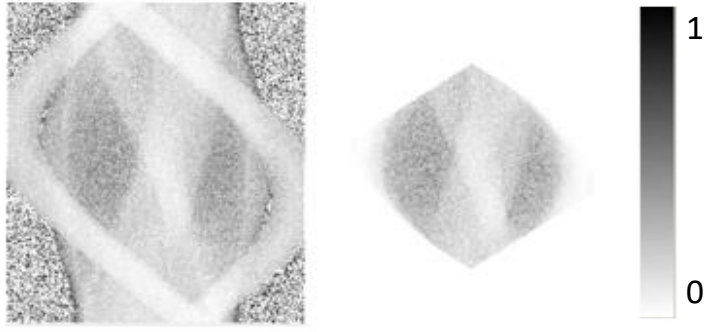

Fig. 11. from left to right: Estimated attenuation factors sinogram for one noise realization of the $\mathrm{W}=50 \mathrm{~cm}, 250 \mathrm{ps}$, closed configuration. Same result for the $\mathrm{W}=50 \mathrm{~cm}, 250 \mathrm{ps}$, open configuration.

the vertical Defrise phantom (figure 9c) all the configurations show similar results, as this phantom is not as sensitive to both truncation and angular limitation due to its orientation with respect to the missing LORs.

For the closed system with the $20 \mathrm{~cm}$ panels, the center of the attenuation image is reconstructed without artefacts. This is because this central region is fully sampled. As mentioned above, reconstruction of such a fully sampled region from truncated tomographic data is called the interior problem [13]. It has been shown that prior knowledge of some of the intensities in that region is sufficient for the problem to have a unique solution inside the interior region [14]. Such prior knowledge was exploited here by imposing the correct attenuation coefficient value for soft tissue, and as a result, artefacts are only observed outside the interior region. In the corresponding activity images, the artefacts outside the interior region are less severe than in the attenuation image, because the activity reconstruction benefits from the TOF resolution along the LORs. For the open system with $20 \mathrm{~cm}$ panels, there is a large angular gap in the data and all projections are severely truncated. As a result, only data from a central part of the object have been acquired, and only that part can be reconstructed. For the activity image, the limited angular artefacts are suppressed by the TOF information along the LORs, but there is no such benefit for the attenuation image. However, the artefacts in the attenuation image do not have to produce attenuation correction errors, the forward projection along the measured LORs will be in agreement with the measurement and enable good attenuation correction.

When the TOF-resolution improves, the activity is reconstructed with lower variance and faster convergence, providing more accurate results in the activity. Because of the faster convergence of the activity, also the estimated attenuation factors converges faster. Nevertheless, the reconstruction of the attenuation image from the estimated attenuation factors is still a non-TOF reconstruction, as the attenuation factors only provides a single value for each LOR. This is the reason why the attenuation images are almost the same for both TOF resolutions.

\section{Simulation with scatter}

Some studies about scatter correction have been done for joint reconstruction algorithms. In [17], [29] the strategy was to generate a first scatter estimate based on two preliminary reconstructions, one for the activity and one for the attenuation in MLAA. In these studies it was shown that alternating the reconstruction of the two images and the estimation of the scatter converged successfully in a few iterations. In the case of the re-scaled MLACF algorithm, a similar technique can be applied as well. Similarly to the MLAA algorithm, the output of the re-scaled MLACF algorithm includes both the activity and the attenuation images as a result of using prior information in the soft tissue region. However, an important difference with the previous studies [17], [29] is that we also consider the case of severe truncation here, which prohibits estimating all the activity and attenuation surrounding the region of interest. More work is required to determine, for different imaging tasks, how much truncation and angular undersampling can be tolerated before their effects on the scatter correction become detrimental.

That analysis of the scatter estimation and correction problem is left for future research, but as a first exploration, the effect of a scatter estimation error on the performance of the re-scaled MLACF algorithm was investigated. For that purpose, re-scaled MLACF reconstructions without scatter correction were computed from scatter-contaminated sinograms. The reconstructed images are obviously not exact, but they have a reasonably good visual quality. At least for the large panels system, they may well be sufficient to initiate the iterative scatter correction procedure mentioned above.

\section{Limitations}

This study has several limitations. The most important one is that only 2D simulations were used, whereas state-of-the art PET systems acquire fully 3D data. In our experience, the findings obtained in 2D simulations are often very predictive of those in fully 3D tomography, but it is clear that the results have to be verified and detailed with more realistic $3 \mathrm{D}$ simulations and real PET measurements. Furthermore, only a single phantom was used, and the phantom had non-zero tracer uptake everywhere. MLACF can only be applied to LORs along which some activity was measured, and for specific tracers with focal uptake, there may be many LORs that have seen no photons. This does not have to be a problem, because for LORs along which no activity was measured, also no attenuation correction is required. However, such LORs will provide no information about the attenuation, creating an even larger missing data problem that may prohibit reconstruction of the attenuation image.

In this study, normalization (i.e. calibration of the LOR sensitivities) was not taken into account. MLACF is robust to normalization errors, it will compensate for them by incorporating them into the MLACF attenuation factors, preventing their propagation into the reconstructed activity image. However, as a result, these errors will propagate into the attenuation image reconstructed from the MLACF attenuation factors, and 


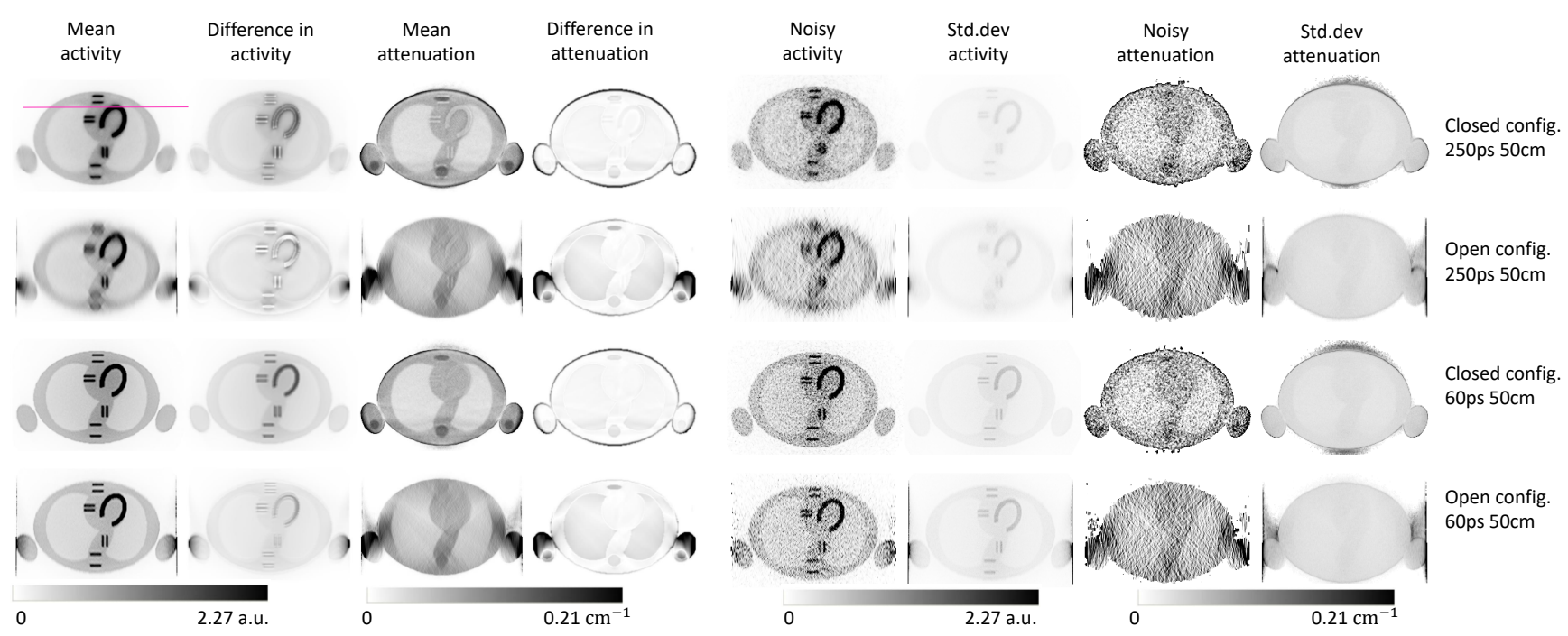

(a)
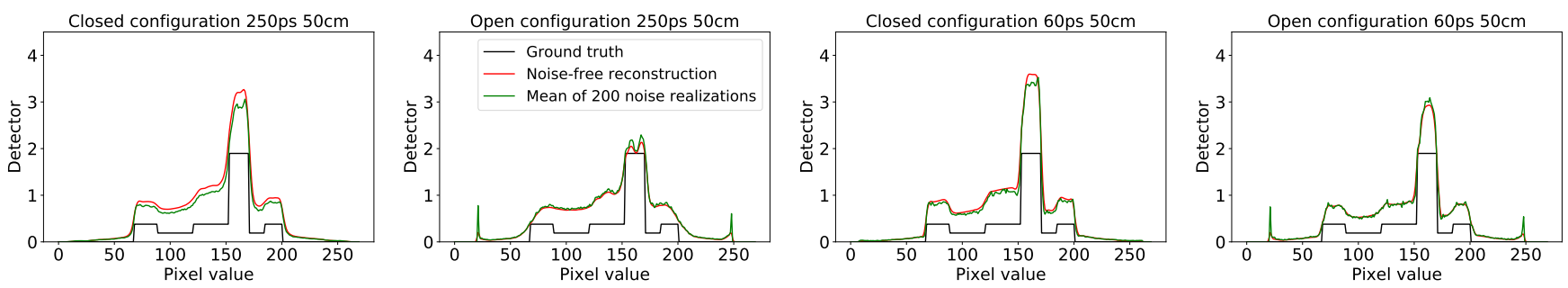

(b)

Fig. 12. Same results as in figure 7 for $W=50 \mathrm{~cm}$ including scatter contribution.

may affect information computed from that image, such as the scale factor and the estimate of the scatter contribution.

\section{CONCLUSION}

In this work, we investigated with simple 2D noise-free and noisy simulations the feasibility of obtaining attenuation corrected images from stand-alone limited angle TOF-PET systems. For the joint estimation of the activity and attenuation images, we proposed a re-scaled MLACF algorithm as a viable and faster alternative for MLAA. This algorithm is faster than MLAA because the reconstruction of the activity and attenuation images are separated, such that incomplete convergence of one image does not affect the other one. A side effect of this approach is that constraints applied during reconstruction of the attenuation image cannot propagate into the activity image. We consider the results promising, warranting further investigation with more sophisticated fully 3D simulations and real PET measurements.

\section{ACKNOWLEDGMENT}

This work was partially supported by the European Research Council (ERC) under the European Unions Horizon 2020 research and innovation program (Grant Agreement No. 695536), by the Research Foundation Flanders (FWO) projects $12 \mathrm{~T} 7118 \mathrm{~N}$ and $\mathrm{G} 062220 \mathrm{~N}$ and by the NIH project 1P41EB017183-01A1.

\section{REFERENCES}

[1] P. Kinahan, D. Townsend, T. Beyer, and D. Sashin, "Attenuation correction for a combined 3D PET/CT scanner," Medical Physics, vol. 25, pp. 2046-53, Nov. 1998.

[2] H. Hao Peng, "Design study of a cardiac-dedicated PET system," Nuclear Instruments and Methods in Physics Research Section A: Accelerators, Spectrometers, Detectors and Associated Equipment, vol. 779, pp. $39-46,2015$.

[3] S. Surti and J. Karp, "Design considerations for a limited angle, dedicated breast, TOF PET scanner," Physics in Medicine and Biology, vol. 53, pp. 2911-21, Jul. 2008.

[4] L. Moliner, A. Gonzalez Martinez, A. Soriano, F. Sánchez, C. Correcher, A. Orero, M. Carles, L. Vidal, J. Barbera, L. Caballero, M. Seimetz, C. Vazquez, and J. Benlloch, "Design and evaluation of the MAMMI dedicated breast PET," Medical Physics, vol. 39, pp. 5393-404, Sep. 2012.

[5] B. Li, Q. Xie, Y. Guo, C. Zeng, S. Wang, R. Zheng, L. Wan, and P. Xiao, "A panel PET with window: design, performance evaluation, and prototype development," IEEE Transactions on Radiation and Plasma Medical Sciences, vol. 1, pp. 310-321, Apr. 2017.

[6] H. Tashima and T. Yamaya, "Proposed helmet PET geometries with addon detectors for high sensitivity brain imaging," Physics in Medicine and Biology, vol. 61, p. 7205-7220, Sep. 2016.

[7] J. Jiang, K. Li, S. Komarov, J. A. O'Sullivan, and Y.-C. Tai, "Feasibility study of a point-of-care positron emission tomography system with interactive imaging capability," Medical Physics, vol. 46, no. 4, pp. 1798-1813, 2019.

[8] A. J. González, F. Sánchez, and J. M. Benlloch, "Organ-dedicated molecular imaging systems," IEEE Transactions on Radiation and Plasma Medical Sciences, vol. 2, no. 5, pp. 388-403, 2018.

[9] H. K. Tuy, "An inversion formula for cone-beam reconstruction," SIAM Journal on Applied Mathematics, vol. 43, no. 3, pp. 546-552, 1983. 


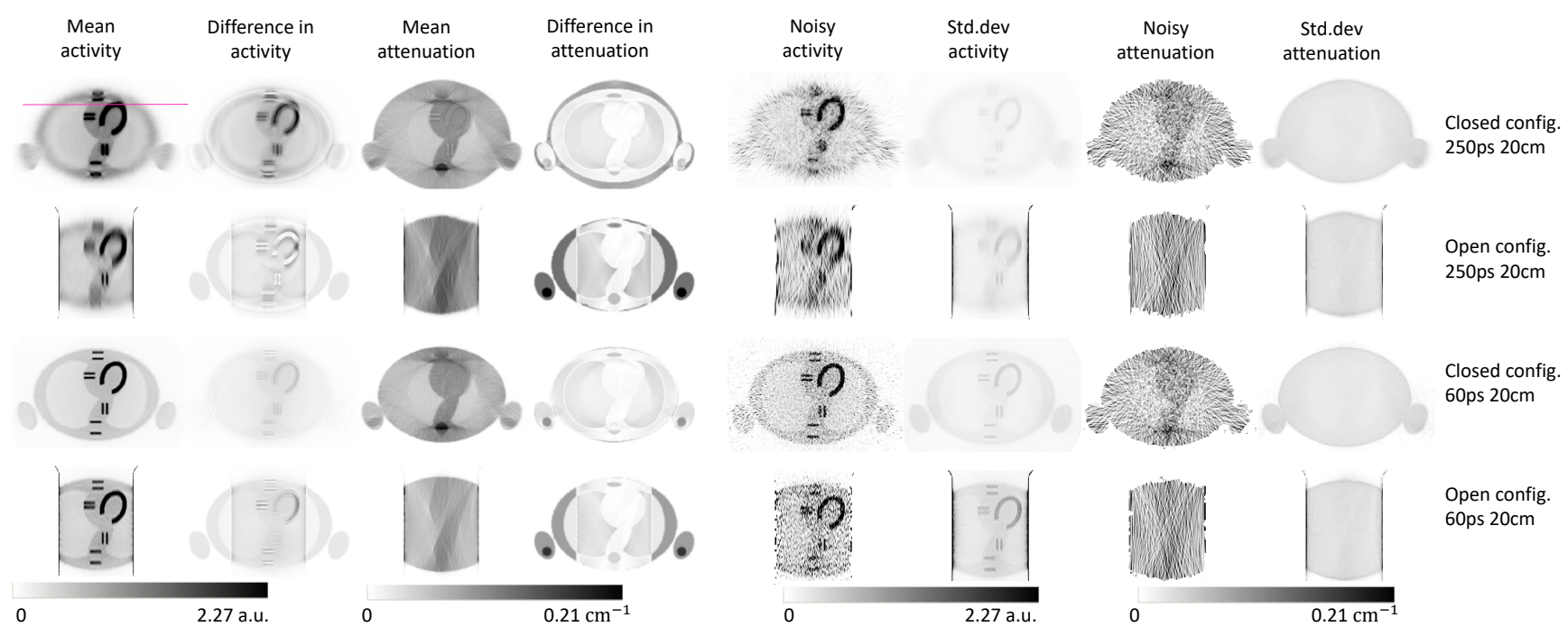

(a)
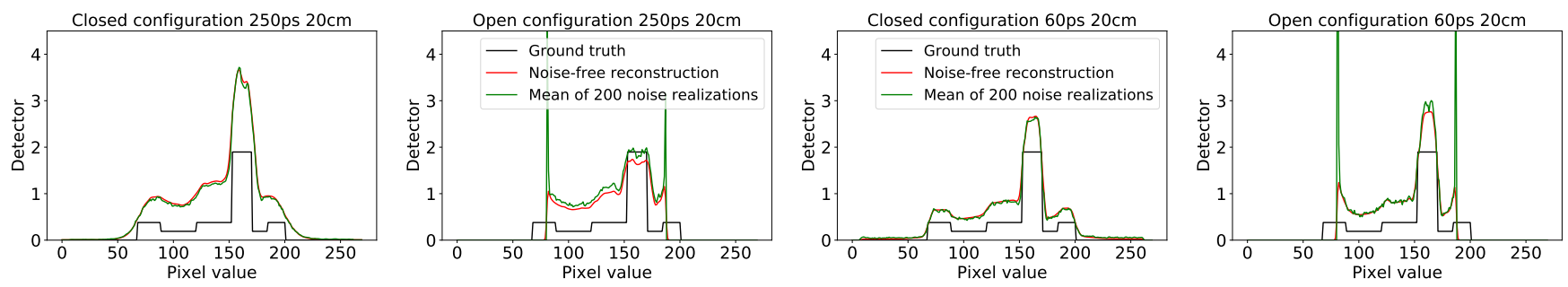

(b)

Fig. 13. Same results as in figure 7 for $W=20 \mathrm{~cm}$ including scatter contribution.

[10] D. V. Finch, "Cone beam reconstruction with sources on a curve," Journal on Applied Mathematics, vol. 45, no. 4, pp. 665-673, 1985.

[11] P. Gravel, Y. Li, and S. Matej, "Effects of TOF resolution models on edge artifacts in PET reconstruction from limited-angle data," IEEE Transactions on Radiation and Plasma Medical Sciences, vol. 4, no. 5, pp. 603-612, 2020.

[12] Y. Li, M. Defrise, S. Matej, and S. Metzler, "Fourier rebinning and consistency equations for time-of-flight PET planograms," Inverse Problems, vol. 32, p. 095004, Sep. 2016.

[13] M. Defrise, F. Noo, R. Clackdoyle, and H. Kudo, "Truncated Hilbert transform and image reconstruction from limited tomographic data," Inverse Problems, vol. 22, p. 1037, May 2006.

[14] M. Courdurier, F. Noo, M. Defrise, and H. Kudo, "Solving the interior problem of computed tomography using a priori knowledge," Inverse Problems, vol. 24, p. 65001, Sep. 2008.

[15] M. Defrise, A. Rezaei, and J. Nuyts, "Time-of-flight PET data determine the attenuation sinogram up to a constant," Physics in Medicine and Biology, vol. 57, pp. 885-99, Feb. 2012.

[16] A. Rezaei, M. Defrise, G. Bal, C. Michel, M. Conti, C. Watson, and J. Nuyts, "Simultaneous reconstruction of activity and attenuation in time-of-flight PET," IEEE Transactions on Medical Imaging, vol. 31, pp. 2224-33, Aug. 2012.

[17] A. Rezaei, G. Schramm, S. Willekens, G. Delso, K. Laere, and J. Nuyts, "A quantitative evaluation of joint activity and attenuation reconstruction in TOF-PET/MR brain imaging," Journal of Nuclear Medicine, vol. 60, p. jnumed.118.220871, Apr. 2019.

[18] S. Ahn, H. Qian, and R. Manjeshwar, "Convergent iterative algorithms for joint reconstruction of activity and attenuation from time-of-flight PET data," IEEE Nuclear Science Symposium Conference Record, pp. 3695-3700, Oct. 2012.

[19] Y. Li, M. Defrise, S. Metzler, and S. Matej, "Transmission-less attenuation estimation from time-of-flight PET histo-images using consistency equations," Physics in Medicine and Biology, vol. 60, p. 6563-6583, Aug. 2015.
[20] S. Ahn, L. S. Cheng, D. Shanbhag, H. Qian, S. Kaushik, F. Jansen, and F. Wiesinger, "Joint estimation of activity and attenuation for PET using pragmatic MR-based prior: Application to clinical TOF PET/MR whole-body data for FDG and non-FDG tracers," Physics in Medicine and Biology, vol. 63, p. 045006, Jan. 2018.

[21] A. Mehranian and H. Zaidi, "Joint estimation of activity and attenuation in whole-body TOF-PET/MRI using constrained gaussian mixture models," IEEE Transactions on Medical Imaging, vol. 34, pp. 1808-21, Mar. 2015.

[22] A. Rezaei, M. Defrise, and J. Nuyts, "ML-reconstruction for TOFPET with simultaneous estimation of the attenuation factors," IEEE Transactions on Medical Imaging, vol. 33, pp. 1563-72, Apr. 2014.

[23] L. A. Shepp and Y. Vardi, "Maximum likelihood reconstruction for emission tomography," IEEE Transactions on Medical Imaging, vol. 1, no. 2, pp. 113-122, 1982.

[24] K. Slambrouck and J. Nuyts, "Metal artifact reduction in computed tomography using local models in an image block-iterative scheme," Medical Physics, vol. 39, pp. 7080-93, Nov. 2012.

[25] Y. Li, S. Matej, and J. Karp, "Practical joint reconstruction of activity and attenuation with autonomous scaling for time-of-flight PET," Physics in Medicine and Biology, Apr. 2020.

[26] S. Ahn, H. Qian, and R. M. Manjeshwar, "Convergent iterative algorithms for joint reconstruction of activity and attenuation from time-offlight pet data," in 2012 IEEE Nuclear Science Symposium and Medical Imaging Conference Record (NSS/MIC), pp. 3695-3700, 2012.

[27] L. Zeng and G. Gullberg, "A study of reconstruction artifacts in cone beam tomography using filtered backprojection and iterative EM algorithms," IEEE Transactions on Nuclear Science, vol. 37, pp. 759767, Apr. 1990.

[28] S. Oliver, L. Moliner, V. Illisie, J. Benlloch, and M. Rodríguez-Álvarez, "Simulation study for designing a dedicated cardiac TOF-PET system," Sensors (Basel), vol. 28, p. 20(5):1311, Feb. 2020.

[29] L. Brusaferri, A. Bousse, E. C. Emond, R. Brown, Y. J. Tsai, D. Atkinson, S. Ourselin, C. C. Watson, B. F. Hutton, S. Arridge, and K. Thiele- 
mans, "Joint activity and attenuation reconstruction from multiple energy window data with photopeak scatter re-estimation in non-TOF 3-D PET,"

IEEE Transactions on Radiation and Plasma Medical Sciences, vol. 4, no. 4, pp. 410-421, 2020. 